\title{
AVALIAÇÃO DAS NOTIFICAÇÕES DE RECEITA PARA ANOREXÍGENOS ENTREGUES A VIGILÂNCIA SANITÁRIA DE MUNICÍPIO DA REGIÃO METROPOLITANA DE RECIFE
}

\author{
EVALUATION OF REVENUE NOTIFICATIONS FOR ANOREXIGENS \\ DELIVERED TO THE SANITARY SURVEILLANCE OF THE \\ METROPOLITAN RECIFE REGION
}

\author{
Clarissa Oliveira Lima Silva ${ }^{1}$ \\ Samantha Mayara Sousa Silva ${ }^{2}$ \\ Adriana Amorim de Farias Leal ${ }^{3}$ \\ Mielle Neiva Santos ${ }^{4}$ \\ Rômulo Moreira dos Santos ${ }^{5}$
}

\begin{abstract}
RESUMO: Objetivo: Analisar as notificações de receitas B2, do Município Goiana, situado na Região Metropolitana do Recife, Pernambuco. Método: Estudo transversal, quantitativo, desenvolvido durante os meses de junho a dezembro de 2019, utilizando-se um formulário padrão para transcrição das informações referentes aos campos prescricionais das receitas, com informações do prescritor, paciente, medicamento e da legibilidade do manuscrito, assim sendo classificadascomo legível, parcialmente legível e ilegível. A pesquisa só foi realizada após aprovação por comitê de ética, recebendo aprovação com a CAAE $n^{\circ}$ 11673219.7.0000.5175. Resultados: Após coletadas informações das 37 Notificações de Receita B2, com as datas de prescrição dentro da faixa temporal do estudo, encontrou-se uma maior prevalência de usuárias do sexo feminino, representando $86,5 \%$ da amostra e, na totalidade, o medicamento foi a sibutramina na dose de $15 \mathrm{mg}$. Com relação a legibilidade dos documentos, foram em sua grande maioria ilegíveis, mesmo este problema sendo combatido desde muito antes das emissões das normativas de controle especial de medicamentos. Conclusão: De acordo com a avaliação dos receituários, identificou-se uma baixa qualidade, no que diz respeito à legibilidade no preenchimento das notificações de receita para a dispensação dos anorexígenos, não fornecendotodos os dados necessários para uma utilização correta e segura dos

\footnotetext{
${ }^{1}$ Graduanda do Curso de Farmácia da Unifacisa.

2 Graduanda do Curso de Farmácia da Unifacisa.

${ }^{3}$ Farmacêutica e Estudante do Programa de Pós-graduação em Saúde Coletiva da UFRN.

${ }_{5}^{4}$ Nutricionista, Mestre em Saúde Pública e Professora do curso de Nutrição da Unifacisa.

5 Farmacêutico, Mestre em Saúde Pública e Professor dos cursos de Farmácia e Nutrição da Unifacisa.
} 
medicamentos. Desta forma, se faz necessário instituir medidas para a diminuição dos possíveis erros de medicação e a melhoria da qualidade das prescrições medicamentosas.

Palavras chave: Notificação de Receita. Anorexígenos. Farmacoepidemiologia.

ABSTRACT: Objective: Analyze the B2 drug prescription of special control, from the Goiana city, located in the Metropolitan Region of Recife, Pernambuco. Method: Cross-sectional, quantitative study, developed during the months of june to december 2019, using a standard form for transcribing information related to the prescriptive fields of prescriptions, with information on the prescriber, patient, medication and the readability of the manuscript, as well as being classified as readable, partially readable and unreadable. The research was only carried out after approval by the ethics committee, receiving approval with CAAE $n^{\circ}$ 11673219.7.0000.5175. Results: After collecting information from the 37 B2 drug prescription of special control, with the prescription dates within the study's time range, a higher prevalence of female users was found, representing $86.5 \%$ of the sample and, in total, the medication was sibutramine at a dose of $15 \mathrm{mg}$. Regarding the legibility of the documents, they were mostly unreadable, even though this problem had been combated since long before the issuance of the special drug control regulations. Conclusion: According to the evaluation of the prescriptions, a low quality was identified, with regard to legibility in filling the prescription notifications for the dispensing ofanorectic agents, not providing all the necessary data for a correct and safe use of the medicines. Thus, it is necessary to institute measures to reduce possible medication errors and improve thequality of drug prescriptions.

Keywords: Drug Prescription of Special Control. Anorexigens. Pharmacoepidemiology. 


\section{INTRODUÇÃO}

A obesidade pode ser definida como o acúmulo excessivo de gordura pelo organismo e, devido as proporções que vem atingindo, é considerada um problema de saúde pública no Brasile em outros países (PEREIRA; FRANCHINI; LANCHA JR, 2003).

Sua incidência, independe de fatores socioeconômicos, traz consequências que afetam diretamente a qualidade de vida dos indivíduos. Segundo a Organização Mundial de Saúde (OMS), cerca de $25 \%$ da população mundial é obesa e destes, um quarto, morrem porconsequências diretas ou indiretas da obesidade (PASSOS, 2005).

A Associação Brasileira para Estudo da Obesidade (ABESO) estima que no Brasil existam, aproximadamente, quatro milhões de obesos mórbidos, equivalente a $4 \%$ da população, sendo a obesidade $30 \%$ das causas de morte e é relacionada às doenças crônicas não transmissíveis (DCNT) como diabetes, hipertensão e outras doenças cardiovasculares (ROMEU; JUSTINO; LIMA, 2008).

O tratamento farmacológico da obesidade está indicado na presença de Índice de Massa Corpórea (IMC) maior que $25 \mathrm{Kg} / \mathrm{m}^{2}$ com outras doenças associadas à este indicador ou em pacientes com IMC maior que $30 \mathrm{Kg} / \mathrm{m}^{2}$, quando da falência do tratamento não farmacológico isoladamente. Pode-se, dividir os fármacos para tratamento da obesidade em três grupos básicos: medicamentos anorexígenos de ação central, medicamentos termogênicos e medicamentos que afetam a absorção de nutrientes (MANCINI, 2004).

Os anorexígenos são fármacos que provocam anorexia, ou seja, redução ou perda de apetite, devendo ser utilizados apenas como adjuvantes no tratamento da obesidade. Sua aplicabilidade não é recomendada como tratamento primário para promover a perda de peso (KOROLKOVAS, 2002).

Contudo, é indispensável uma reflexão da necessidade de utilização e prescrição desses fármacos, pois são parcialmente efetivos, possuem um alto 
número de efeitos colaterais, além de rápida instalação de dependência e tolerância (BEHAR, 2002).

A avaliação da prescrição e dispensação de fármacos anorexígenos é imprescindível, visto que "o Brasil é um dos países onde mais cresce o consumo destes medicamentos" (JIPE, 2005). A respeito desses medicamentos e/ou associações que eles os contenham, é de bastanterelevância as informações, pois o seu uso de forma indiscriminada acarreta problemas de seguridade e eficácia.

Anfetaminas e seus derivados utilizados como anorexígeno ou no distúrbio de hiperatividade em crianças, têm sua comercialização sujeita às exigências da Portaria $n^{\circ} 344$ de 12 de maio de 1998, da Secretaria da Vigilância Sanitária (BRASIL, 2005). O uso indiscriminado e excessivo de fármacos levou a ANVISA a dispor de instrumentos regulatóriospara proteger a saúde da coletividade e ter maior controle do comércio de medicamentos psicotrópicos, inclusive os anorexígenos.

Com intuito de aumentar as informações farmacoepidemiológicas da utilização dos anorexígenos de ação central, regulados pelas normativas da Secretaria de Vigilância Sanitária (SVS) presentes na Portaria SVS 344/1998 e Portaria SVS 06/1999 (BRASIL, 1998; BRASIL, 1999), assim como nas atualizações contidas nas resoluções RDC 50/2014 e RDC 133/2016 da Agência Nacional de Vigilância Sanitária (ANVISA) (BRASIL, 2014; BRASIL, 2016), este estudo avaliou as prescrições exigidas para este tipo de medicamento.

\section{MÉTODO}

O trabalho foi caracterizado como um estudo de prevalência, quantitativo e descritivo, sendo realizado no Município de Goiana, situado na Região Metropolitana do Recife, Pernambuco, durante os meses de junho a dezembro de 2019.

Para a pesquisa, foram usados como fonte de dados os Relatórios Mensais de Notificação de Receita (RMNR) B2, contendo as Notificações de Receita (NR) B2, específicas para anorexígenos e entregues mensalmente a Vigilância Sanitária municipal. Ademais, as NR consultadas apresentaram datas de prescrição entre 
junho de 2018 a junho de 2019. A coleta dedados foi realizada no Departamento de Vigilância Sanitária do município citado após o recebimento e conferência dos RMNR B2, utilizando-se um formulário padrão para transcrição das informações referentes aos campos prescricionais das NRs, com informações do prescritor, paciente, medicamento e da legibilidade do manuscrito, conforme a classificação proposta por Rosa et al. (2009), onde cada palavra deveria ser avaliada separadamente, evitando interpretação ou dedução, assim, a prescrição deveria ser entendida e não interpretada, classificando-as como Legível (leitura fácil, sem problema ou perda de tempo para compreensão do que está escrito ou com dúvidas para o entendimento de todas as palavras, siglas e números), Parcialmente legível (leitura com dificuldade, utilizando-se um maior tempo para compreensão do manuscrito) e llegível (quando, independentemente do tempo da análise,foi impossível de entender o que estava escrito).

O estudo obedeceu a Resolução n $466 / 2012$ do Conselho Nacional de Saúde e só foi desenvolvido após a aprovação pelo Comitê de Ética em Pesquisa do Centro de Ensino Superior e Desenvolvimento (CESED), recebendo a CAAE $n^{\circ}$ 11673219.7.0000.5175.

\section{RESULTADOS}

Foram coletadas informações de 37 Notificações de Receita B2 com as datas de prescrição dentro da faixa temporal do estudo. As NR prescritas para usuárias do sexo feminino representaram $86,5 \%$ da amostra e, na totalidade, o medicamento continha a sibutramina na dose de $15 \mathrm{mg}$. As demais informações dos campos prescricionais se encontram na Tabela 1. 
Tabela 1. Distribuição da frequência das variáveis prescricionais das $N R^{*} B 2$ avaliadas pelo estudo.

\begin{tabular}{|c|c|c|}
\hline Variáveis & $\mathbf{N}$ & $\%$ \\
\hline Sexo & 08 & 21,63 \\
\hline Feminino & 32 & 86,50 \\
\hline Masculino & 05 & 13,50 \\
\hline \multicolumn{3}{|l|}{ Procedência do Paciente } \\
\hline Pernambuco & 37 & 100,00 \\
\hline Outro Estado & 00 & 0,00 \\
\hline \multicolumn{3}{|l|}{ NR própria do prescritor } \\
\hline Sim & 16 & 43,24 \\
\hline Não & 21 & 56,76 \\
\hline \multicolumn{3}{|l|}{ Especialidade Médica } \\
\hline Endocrinologista & 30 & 81,08 \\
\hline Clínico Geral & 07 & 18,92 \\
\hline \multicolumn{3}{|l|}{ Medicamento e Dose } \\
\hline Sibutramina $15 \mathrm{mg}$ & 37 & 37,00 \\
\hline \multicolumn{3}{|l|}{ Posologia } \\
\hline 1 comprimido ao dia & 36 & 97,30 \\
\hline 2 comprimidos ao dia & 01 & 2.70 \\
\hline \multicolumn{3}{|l|}{ Dispensação Farmacêutica } \\
\hline Sim & 18 & 48.65 \\
\hline Não & 19 & 51,35 \\
\hline \multicolumn{3}{|l|}{ Quantidade Dispensada } \\
\hline 1 caixa & 15 & 40,54 \\
\hline 2 caixas & 22 & 59,46 \\
\hline \multicolumn{3}{|l|}{ Tipo do medicamento prescrito } \\
\hline Referência & 08 & 29,59 \\
\hline Genérico & 29 & 72,41 \\
\hline \multicolumn{3}{|l|}{ Tipo do medicamento dispensado } \\
\hline Referência & 14 & 37,85 \\
\hline Genérico & 18 & 48,65 \\
\hline Similar & 05 & 13,50 \\
\hline \multicolumn{3}{|l|}{ Identificação do laboratório } \\
\hline Sim & 31 & 83,78 \\
\hline Não & 06 & 16,22 \\
\hline TOTAL & 37 & 100,00 \\
\hline
\end{tabular}

Fonte: Dados do estudo, 2019 - *NR = Notificação de Receita.

Todas as NR B2 estavam acompanhadas do Termo de Responsabilidade do Prescritor, porém, em $04(10,81 \%)$ termos não constava a justificativa da prescrição, 
em 05 (13,50\%) não estavam as informações de idade ou sexo e sobre a anuência dos usuários, 02 (05,40\%) não estavam assinados.

Com relação a legibilidade dos campos prescricionais, as informações foram classificadas de acordo com o descrito na Tabela 2, não sendo encontradas NR B2 sem algum campo escrito pelo profissional prescritor.

Tabela 2. Análise quanto à legibilidade das $37 \mathrm{NR}^{\star} \mathrm{B} 2$ consultadas.

\begin{tabular}{|c|c|c|}
\hline Variáveis & $\mathbf{N}$ & $\%$ \\
\hline \multicolumn{3}{|l|}{ Dados do Paciente } \\
\hline Legível & 08 & 21,63 \\
\hline Parcialmente legível & 08 & 21,63 \\
\hline llegível & 21 & 56,74 \\
\hline \multicolumn{3}{|l|}{ Dados do Prescritor } \\
\hline Legível & 04 & 10,81 \\
\hline Parcialmente legível & 14 & 37,84 \\
\hline llegível & 19 & 51,35 \\
\hline \multicolumn{3}{|l|}{ Nome do Medicamento } \\
\hline Legível & 10 & 27,03 \\
\hline Parcialmente legível & 18 & 27,03 \\
\hline llegível & 09 & 45,94 \\
\hline \multicolumn{3}{|l|}{ Dose } \\
\hline Legível & 11 & 29,73 \\
\hline Parcialmente legível & 20 & 54,05 \\
\hline llegível & 06 & 16,22 \\
\hline \multicolumn{3}{|l|}{ Posologia } \\
\hline Legível & 03 & 08,11 \\
\hline Parcialmente legível & 12 & 32,43 \\
\hline llegível & 22 & 59,46 \\
\hline TOTAL & 37 & 37,00 \\
\hline
\end{tabular}

Fonte: Dados do estudo, 2019 - NR = Notificação de Receita.

\section{DISCUSSÃo}

Os anorexígenos são drogas ilícitas, com comércio controlado e dependente de emissão de receita especial por parte do médico ao farmacêutico. O controle especial é um meio que a saúde pública possui para evitar o uso indevido destes 
medicamentos e garantir seu uso às necessidades de ordem médica (MALTA JÚNIOR, 2003). Devido a isso, a orientação dos profissionais é ainda mais importante para que o uso destes medicamentos não leve a ineficáciaa longo prazo e ao reganho de peso.

De acordo com a RDC 50/2014 da ANVISA, que dispõe sobre as medidas de controle de comercialização, prescrição e dispensação de medicamentos que contenham as substâncias anfepramona, femproporex, mazindol e sibutramina diz que, além da NR B2, o profissional médico deve disponibilizar o Termo de Responsabilidade do Prescritor, para acompanhar a prescrição e ser preenchido em três vias: uma via deve ser arquivada no prontuário do paciente, a outra na farmácia ou drogaria dispensadora e uma via mantida com o usuário (BRASIL, 2014).

Esse termo, que é assinado pelo médico e paciente, e indica casos em que o uso do medicamento não dever ser feito, bem como os riscos a que o usuário está sujeito, além de informar ao paciente a importância do uso concomitante com uma alimentação saudável e prática de atividades físicas. Contrariando as exigências desta resolução, havia termos com preenchimento incompleto, o que pode dificultar a rastreabilidade dos usuários em caso de problemas associados da prescrição ao uso do produto.

Ainda, todas as justificativas citadas foram apenas "obesidade", sem classificar essa condição pelo IMC como obesidade I, II ou III. Agravando este fato, em quase onze por centonão estava com essa informação preenchida.

Também obriga o médico e o farmacêutico a notificarem no VigiMed eventos adversos a medicamentos que contenham a substância sibutramina, seus sais e isômeros, bem como intermediários, sendo de notificação compulsória ao Sistema Nacional de Vigilância Sanitária (ANVISA, 2011).

Dos receituários analisados, em relação ao número de comprimidos prescritos, foi observada alta frequência na dispensação de 2 caixas com 30 comprimidos em 22 receituários, sendo está a quantidade padronizada pelas indústrias farmacêuticas. De acordo com a RDC 133/2016 da ANVISA (BRASIL, 2016), a quantidade prescrita de sibutramina é para tratamento máximo de sessenta dias, assim, não foram encontrados erros de prescrição ou dispensação. 
Analisando os medicamentos dispensados, foi observado que na maioria dos receituários $(48,65 \%)$ foram referentes ao medicamento genérico, seguidos de 14 (37,85\%) dereferência e 05 (13,50\%) similares. Os medicamentos de referência são mais caros que os outros, porém os médicos priorizam o uso destes, justificando a grande quantidade de dispensação em drogarias. Conforme a Lei Federal 9.787/99 é permitida a intercambialidade pelo profissional farmacêutico entre medicamentos de referência pelos genéricos. Segundo dados da Associação Brasileira das Indústrias de Medicamentos Genéricos, as apresentações de medicamentos genéricos disponíveis no mercado brasileiro atendem a mais de $60 \%$ das necessidades de prescrições médicas. Entretanto, há uma preferência dos medicamentos de referência devido ao estigma social imposto e preconceito com medicamentos que podem ser intercambiáveis (BLATT et al., 2012).

No item de dispensação farmacêutica, estiveram ausentes, em mais da metade das NR B2 analisadas, o carimbo e assinatura do profissional farmacêutico, indicando uma exigência negligenciada pelos responsáveis técnicos das unidades de saúde, contrariando, além das normas de controle especial de medicamentos, as orientações do Código de Ética Farmacêutica e de Boas Práticas de dispensação (CFF, 2014). Também é de responsabilidade do farmacêuticoo registro da quantidade dispensada, assim como é de responsabilidade do prescritor registrar a quantidade prescrita.

Observou-se a predominância do uso de inibidores do apetite entre pacientes do sexo feminino, mostrando as mulheres como grandes consumidoras desses fármacos, corroborando o fato da exigência da magreza como fator determinante da beleza. Um impedimento do levantamento dessas informações é que em nenhum documento entregue às farmácias constamo IMC das usuárias, além da declaração do diagnóstico de obesidade presente no Termo de Responsabilidade do Prescritor (FREITAS et al., 2010; GONÇALVES et al., 2020).

Com relação a legibilidade dos documentos, foram em sua grande maioria de forma ilegível. Este problema já é combatido desde muito antes das emissões das normativas de controle especial de medicamentos, datando orientações já na década de setenta do século passado, com a Lei Federal 5.991 de 1973 (BRASIL, 1973). Todas as diretrizes para prescrição de medicamentos proíbem o uso de 
rasuras, siglas ou emendas que dificultem o entendimento e possibilitem erro nas etapas seguintes do uso de medicamentos, aumentando a possibilidade de uso inadequado e com potenciais prejuízos à qualidade de vida dos usuários.

Os dados apresentados corroboram com a necessidade de educação continuada dos profissionais prescritores e dispensadores e, também, a adoção de medidas para a melhoria da legibilidade de informações sobre o medicamento e seu modo de uso Além dos possíveis erros de medicação, a dificuldade na leitura de qualquer receita pode prejudicar a adesão aotratamento pelo paciente (OLIVEIRA, SANTOS, LEITE, 2015).

\section{CONCLUSÃO}

A partir dos resultados encontrados neste estudo, identificou-se uma baixa qualidade, no que diz respeito à legibilidade no preenchimento dos receituários para a dispensação dos anorexígenos, que não fornecem todas as informações necessárias para uma utilização correta e segura dos medicamentos.

Desta forma, se faz necessário instituir medidas para a diminuição dos possíveis erros de medicação e a melhoria da qualidade das prescrições medicamentosas. A sensibilização dos prescritores sobre a importância de uma prescrição clara, completa e precisa para que se possa imprimir segurança no ciclo do uso do medicamento, ressaltando a necessidade da implementação de estratégias que reforcem o benefício de uma prescrição correta. Dentre as medidas, recomendam-se simplificação e padronização dos processos de prescrição, dispensação e administração de medicamentos, implementação de prescrição por sistema computadorizado em todas as clínicas, utilização de software farmacêutico como fonte de informação sobre medicamentos e checagem de prescrições.

Destaca-se também a necessidade da adoção de medidas educativas para prescritores, frente aos aspectos éticos que norteiam a prescrição e dispensação dessa classe de medicamentos, a fim de evitar o uso indiscriminado a partir de um diagnóstico e prescrição equivocada. 


\section{REFERÊNCIAS BIBLIOGRÁFICAS}

BEHAR, R. Anorexígenos: indicações e interações. Revista Chilena de Neuropsiquiatria, v. 40, p. 21-36, 2002.

BLATT CR, TRAUTHMAN SC, SCHMIDT EH, MARCHESAN S, SILVA LM, MARTINS JL. Conhecimento popular e utilização dos medicamentos genéricos na população do município de Tubarão, SC. Ciênc. saúde coletiva. 2012.

BRASIL. Agência Nacional de Vigilância Sanitária. RDC n 52, de 6 de outubro de 2011. Brasília, DF: 2011.

BRASIL. Ministério da Saúde. Secretária de Vigilância Sanitária. Portaria n. 344, de 12 de maio de 1998. Aprova o Regulamento técnico sobre substâncias e medicamentos sujeitos a controle especial. Brasília, DF: 1998.

CONSELHO FEDERAL DE FARMÁCIA. RDC 596, de 21 de fevereiro de 2014. Dispõe sobre o Código de Ética Farmacêutica, o Código de Processo Ético e estabelece as infrações e as regras de aplicação das sanções disciplinares. Brasília: CFF, 2014.

FREITAS, C. M. S. M.; LIMA, R. B. T.; COSTA, A. F.; LUCENA FILHO, A. O padrão de beleza corporal sobre o corpo feminino mediante o IMC. Rev. Bras. Educ. Fís. Esporte. v. 24,n. 3, p.389-404, 2010.

GONÇALVES, F. T.; MENEGON, V. G. S.; OLIVEIRA, M. M. S.; SILVA, R. R.; CARNEIRO, M. S. LEMOS, A. V. L. et al. Imagem corporal feminina e os efeitos sobre a saúde mental: uma revisão bibliográfica sobre a intersecção entre gênero, raça e classe. REAS.v. 39, Sup. e2194, 2020.

JIFE, Boletim Informativo da Junta Internacional de Fiscalização de Entorpecentes. Anorexígenos. 2005.

KOROLKOVAS, A. Metabolismo e Nutrição. Dicionário Farmacêutico Guanabara. Guanabara Koogan, Rio de Janeiro, 2002.

MALTA JÙNIOR, A. Avaliação da dispensação de benzodiazepínicos em farmácia comercialde Juazeiro do Norte - CE. Infarma, Fortaleza, v.15, p. 50-2, 2003.

MANCINI, M.C. Obesidade - como diagnosticar e tratar. Rev. Bras. Med., v.61, Ed. Especial, 2004.

OLIVEIRA, C. S.; SANTOS, A. S.; LEITE, I. C. G. Avaliação da qualidade das prescrições médicas da farmácia municipal de Catalão - Goiás. Rev Med Minas Gerais. v. 25, n. 4, p. 556$561,2015$.

PASSOS, W.V. Obesidade: visão da nutricionista. Portal de ginecologia, 2002.

PEREIRA L.O.; FRANCHINI R.P.; LANCHA J.R.. Obesidade: hábitos nutricionais, sedentarismo e resistência à insulina. Arq Bras Endocrinol Metab. v.47, n.2, p.111-127. 2003.

ROMEU, G. A.; JUSTINO, P. F. C.; LIMA, A. M. A.. Perfil da prescrição e dispensação de fármacos anorexígenos em Fortaleza - CE. Infarma, Fortaleza, v. 20, n. 7/8, p. 32-37, 2008.

ROSA, M. B.; PERINI, E.; NACLETO, T. A.; NEIVA, H. M.; BOGUTCHI, T. Erros na prescrição hospitalar de medicamentos potencialmente perigosos. Rev Saúde Pública, v. 43, p.490-98, 2009. 